Article

\title{
Enhancement of $\mathrm{NH}_{3}$ Gas Sensitivity at Room Temperature by Carbon Nanotube-Based Sensor Coated with Co Nanoparticles
}

\author{
Lich Quang Nguyen ${ }^{1}$, Pho Quoc Phan ${ }^{1}$, Huyen Ngoc Duong ${ }^{1}$, Chien Duc Nguyen ${ }^{1,2}$ and \\ Lam Huu Nguyen ${ }^{1,2, *}$
}

1 School of Engineering Physics, Hanoi University of Science and Technology, 1 Dai Co Viet Rd, Hanoi 10000, Vietnam; E-Mails: nqlich1501@yahoo.com (L.Q.N.); pq_pho@yahoo.com (P.Q.P.); huyen.duongngoc@hust.edu.vn (H.N.D.); chien.nguyenduc@hust.edu.vn (C.D.N.)

2 Advanced Institute of Science and Technology, Hanoi University of Science and Technology, 1 Dai Co Viet Rd, Hanoi 10000, Vietnam

* Author to whom correspondence should be addressed; E-Mail: lam.nguyenhuu@hust.edu.vn; Tel.: +84-4-3868-2540; Fax: +84-4-3869-3498.

Received: 27 November 2012; in revised form: 25 January 2013 / Accepted: 29 January 2013 / Published: 30 January 2013

\begin{abstract}
Multi-walled carbon nanotube (MWCNT) film has been fabricated onto Pt-patterned alumina substrates using the chemical vapor deposition method for $\mathrm{NH}_{3}$ gas sensing applications. The MWCNT-based sensor is sensitive to $\mathrm{NH}_{3}$ gas at room temperature. Nanoclusters of Co catalysts have been sputtered on the surface of the MWCNT film to enhance gas sensitivity with respect to unfunctionalized CNT films. The gas sensitivity of Co-functionalized MWCNT-based gas sensors is thus significantly improved. The sensor exhibits good repeatability and high selectivity towards $\mathrm{NH}_{3}$, compared with alcohol and LPG.
\end{abstract}

Keywords: multi-walled carbon nanotube; $\mathrm{CVD}$; sensor; $\mathrm{NH}_{3}$; catalyst

\section{Introduction}

New materials and treatment processes for gas sensing applications that can detect gaseous molecules have attracted increasing attention for several years. Gas sensing studies aim to create an electronic nose that can detect each kind of gas which is present in ambient atmosphere at low concentration levels, with sufficient sensitivity, selectivity, and reproducibility. Several types of gases, such as liquefied petroleum gas (LPG), alcohol, $\mathrm{H}_{2}, \mathrm{NH}_{3}, \mathrm{NO}_{2}$, and $\mathrm{CO}_{2}$, are toxic, harmful, or 
flammable. Among these gases, $\mathrm{NH}_{3}$ is the most common and is used in food processing, environmental remediation, agriculture, and medical diagnostics.

$\mathrm{NH}_{3}$ gas sensors have been developed based on semiconductor metal oxides, such as $\mathrm{SnO}_{2}$ and $\mathrm{ZnO}$, which require high operational temperatures that range from $300{ }^{\circ} \mathrm{C}$ to $400{ }^{\circ} \mathrm{C}[1,2]$. To save energy and reduce the working temperature, researchers are currently looking for new materials to replace these materials. In this aspect, carbon nanotubes (CNTs) hold great promise because of their many advantages. For instance, CNTs function effectively at room temperature and have a high surface per weight ratio. To apply CNTs on $\mathrm{NH}_{3}$ sensing application, several gas sensor models based on CNTs, such as gas ionization sensor [3], capacitive sensor [4,5], CNT-field effect transistor [6], resonant-circuit sensor [7], and resistance sensor [8], have been developed. The resistance sensor model is a simpler approach than other models, which are used to fabricate and set up measurement systems.

In this paper, multi-walled carbon nanotube (MWCNT) films were synthesized using the chemical vapor deposition (CVD) method, in which $\mathrm{Ni}$ was used as a catalyst on an alumina substrate. The MWCNTs were purified by sintering at $400{ }^{\circ} \mathrm{C}$ in dry air. The size and structure of the MWCNTs were observed by field emission scanning electron microscopy (FESEM). $\mathrm{NH}_{3}$ sensitivity of the MWCNT films was investigated at room temperature by using a static measurement system. Co-nanoparticle catalyst was coated on the MWCNTs to enhance CNT sensitivity, thereby promoting $\mathrm{NH}_{3}$ gas interaction.

\section{Experimental}

In the resistance sensor model, the MWCNT film was synthesized in the area between two Pt electrodes, which were fabricated on an alumina substrate by using a screen printing technique. Before growing the MWCNT film, a Ni catalyst layer was sputtered on this surface. The thickness of the catalyst layer was approximately $8 \mathrm{~nm}$. The substrate was then placed into the reaction chamber. The MWCNT film was synthesized based on the CVD method at $725^{\circ} \mathrm{C}$ for $30 \mathrm{~min}$ with acetylene $\left(\mathrm{C}_{2} \mathrm{H}_{2}\right)$ as the gas source. Inert $\mathrm{N}_{2}$ gas was used to protect the CNTs from oxygen in the atmosphere. After the growth process, the CNTs were cooled naturally in the reaction chamber, always with the inert $\mathrm{N}_{2}$ gas flow.

The purification process was also conducted in the reaction chamber. The sample was heated to $400{ }^{\circ} \mathrm{C}$ in dry air to reduce the amorphous carbonaceous material. Oxygen in the atmosphere acts as an oxidizing agent. The morphology of the obtained CNTs was characterized by FESEM. The CNT-based sensor was then used to determine $\mathrm{NH}_{3}$ sensitivity by using a static measurement system with a Keithley 6487 picoammeter/voltage source. The gas concentration in the static system was determined using the Canadian BW Gas Alert. A $2 \mathrm{~nm}$ thick Co layer was coated on the MWCNT film to enhance the sensing performance of MWCNT to $\mathrm{NH}_{3}$ gas. The metal nanoparticle catalyst was used to increase the sensitivity and the selectivity of the CNT-based sensor.

\section{Results and Discussion}

The diagram of a resistance-type gas sensor is shown in Figure 1(a). The CNT layer was grown on a defined area with two Pt electrodes. Figure 1(b-d) shows the SEM images of the samples synthesized at $725^{\circ} \mathrm{C}$. Before the samples were obtained, the samples were sintered at $400{ }^{\circ} \mathrm{C}$ for $30 \mathrm{~min}$ in dry air to remove the amorphous carbonaceous material. It is noted that this sintering procedure has been often used to reduce number of impurities and/or defects [9]. The CNTs are very uniform and have an 
average diameter of approximately $30 \mathrm{~nm}$. The obtained CNTs are multi-walled based on the growth condition and their size, as well as Raman study (not shown here). During the growth process, when the CNTs are long enough, they interact with each others, thereby creating a CNT film, which resembles as a carpet. The FESEM images also show that the CNTs were only formed on the area between the Pt electrodes Figure 1(b). The CNTs are not present in the Pt electrodes because of the difference in the interactions between the metal catalyst and the surfaces.

Figure 1. Diagram of the growth of carbon nanotubes (CNTs) on the alumina substrate with Pt electrodes (a); CNT formation between two electrodes (b); Contact between CNTs and electrode (c); CNTs on the alumina surface $(\mathbf{d})$.

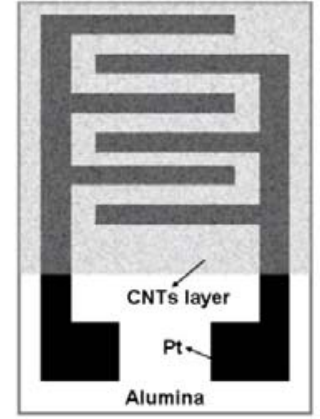

a)

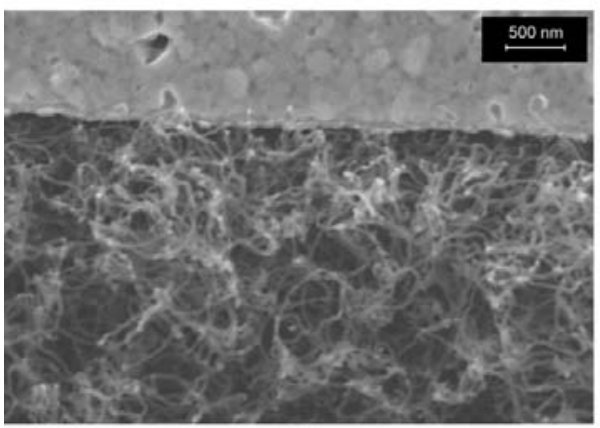

c)

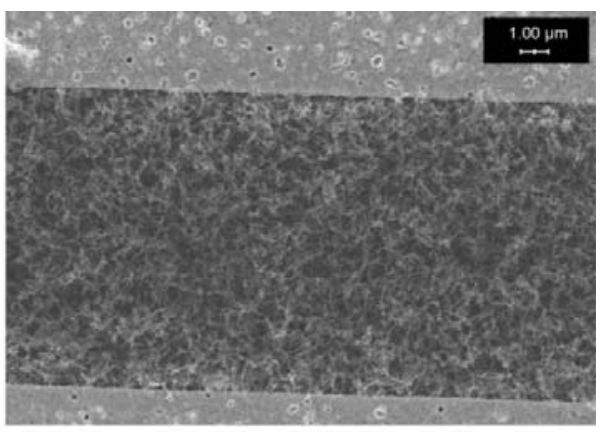

b)

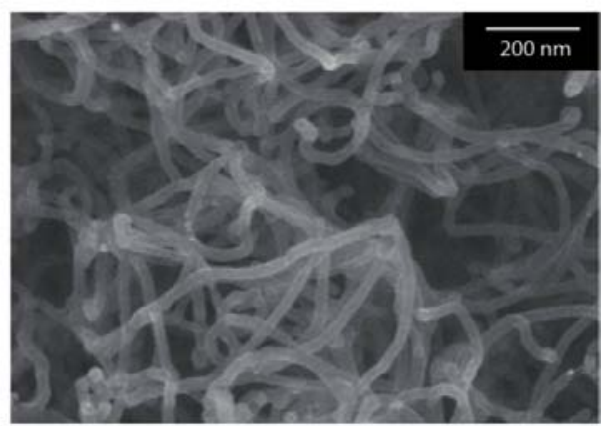

d)

The CNT growth process mainly occurs through the vapor-liquid-solid mechanism. In this case, CNT growth is supported by catalytic particles and the size of CNT depends on the dimension of these catalytic particles. The catalyst particles play an important role in the growth process. Only suitable catalytic particles can promote CNT formation. Once on the screen printed Pt electrode surface, the catalyst can then penetrate into the Pt electrodes. The Pt layer prevents the metal catalyst atoms from interacting with one another to form suitable particles. In contrast, an alumina surface found in the area between the electrodes helps the catalyst atoms to move and form catalytic particles with suitable sizes. Afterward, CNTs can be grown. The density of CNTs is very high; thus, CNTs can easily bridge the electrodes, thereby creating a contact between the MWCNT film and the electrodes Figure 1(c).

We assumed that the obtained MWCNT behaved as both metallic and semiconducting materials, depending on the synthesis of CNTs, their diameter, chirality as well as the defect and adsorbed gas. Only small amount of semiconducting CNTs formed among predominant conducting CNTs during growing process [10-13]. When the $\mathrm{NH}_{3}$ gas adsorbed on the sidewalls, edges or tube ends, the electrical conductivity of the MWCNTs is changed $[14,15]$. The sensing mechanism of CNT-based gas 
sensors involves charge transfer, which occurs during the interaction of gas molecules with the CNT surface. This interaction modifies the conductivity of CNTs [8-17]. The resistance of the MWCNT film increases when exposed to $\mathrm{NH}_{3}$ molecules, which are electron-donating molecules. This phenomenon likely occurs through the interaction of $\mathrm{NH}_{3}$ molecules with the carbon molecules on the CNT sidewalls. Therefore, electrons are transferred from $\mathrm{NH}_{3}$ molecules to CNTs. $\mathrm{NH}_{3}$ molecules donate electrons to form a space charge region (depletion region) on the semiconducting CNT surface. This depletion region decreases the holes transport, thereby increasing the electrical resistance of CNTs.

In our gas sensing measurement, the sensor response is defined as the relative resistance change: Response (\%) $\left.=\left[\left(R_{\text {gas }}-R_{\text {air }}\right) / R_{\text {air }}\right)\right] \times 100 \%$, where $R_{\text {gas }}$ and $R_{\text {air }}$ are the resistance of the MWCNT film in $\mathrm{NH}_{3}$ gas environment and in dry air, respectively. Figure 2(a,b) shows the sensor response against the measurement time in low and high $\mathrm{NH}_{3}$ gas concentrations, respectively. The CNT sensor responds to $\mathrm{NH}_{3}$ at room temperature, i.e., when the $\mathrm{NH}_{3}$ gas is adsorbed on the CNT film, the sensor becomes immediately sensitive to the gas. The response then reaches a saturated value at a defined gas concentration.

Figure 2. Sensor responses to $\mathrm{NH}_{3}$ gas at a low concentration $(<100 \mathrm{ppm})$ (a) and high concentration (up to $800 \mathrm{ppm}$ ) (b). Dependence of the sensor responses on $\mathrm{NH}_{3}$ gas concentrations shows two corresponding linear regions $(\mathbf{c})$.

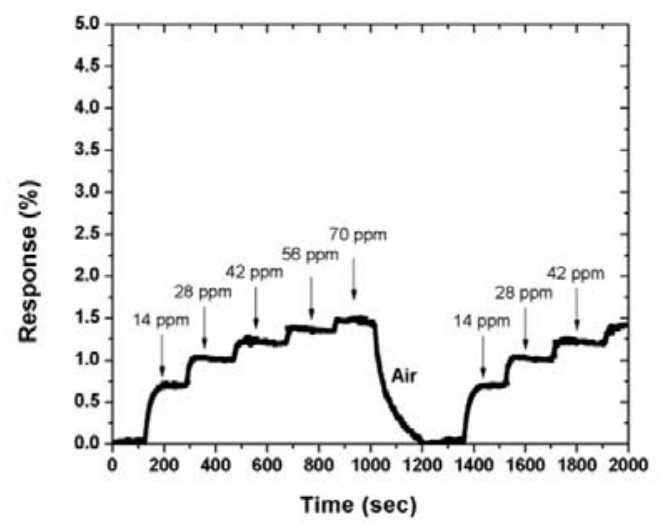

a)

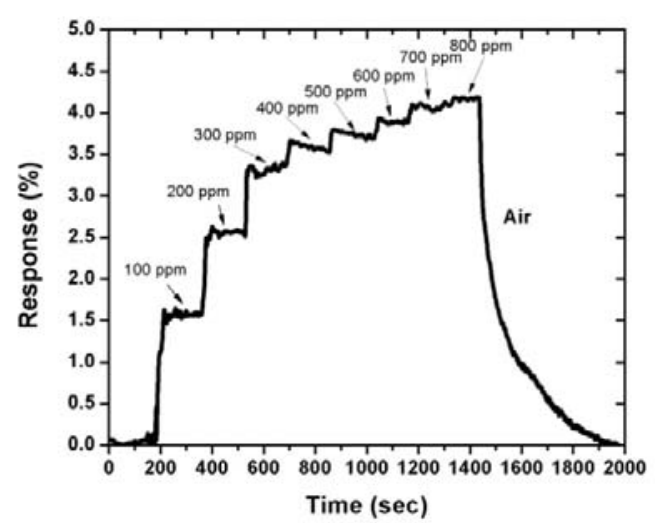

b)

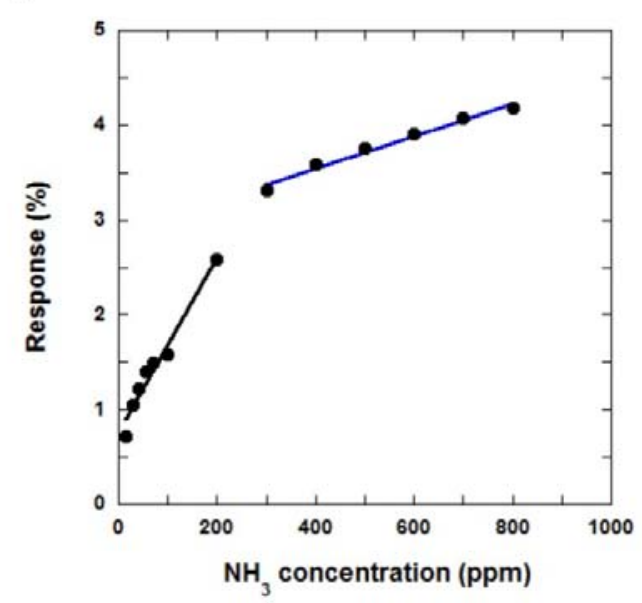

c) 
At low $\mathrm{NH}_{3}$ concentrations, the response of the sensor is increased slightly as the gas concentration is increased. For instance, when the gas concentration was increased to $70 \mathrm{ppm}$, at a step of $14 \mathrm{ppm}$, the response increased and was maintained at approximately $1.5 \%$. In contrast, the response decreased to approximately $0 \%$ when the sensor was exposed to air Figure 2(a). The recovery time in this case was approximately $200 \mathrm{~s}$. The measurements were also repeated by injecting $\mathrm{NH}_{3}$ gas into the system at the same concentrations. This result demonstrated that the CNT film was stable during the measurement process. We also determined the sensor response at high $\mathrm{NH}_{3}$ gas concentrations of $100 \mathrm{ppm}$ to $800 \mathrm{ppm}$. Figure 2(b) shows the responses of the $\mathrm{CNT}$ film to $\mathrm{NH}_{3}$ gas. The response time of the sensor is approximately $30 \mathrm{~s}$ to $50 \mathrm{~s}$ and the recovery time was approximately $500 \mathrm{~s}$ at the $\mathrm{NH}_{3}$ gas concentration of $800 \mathrm{ppm}$.

The relationship between the responses of the sensor and $\mathrm{NH}_{3}$ concentration is shown in Figure 2(c), which can be divided into two linear regions: the low concentration region and the high concentration region. The sensitivity increases more rapidly in the low concentration region than that in the high concentration region. Therefore, the fitting curve of the former presented a bigger slope compared with that of the latter. This observation occurs because of CNT film structure, in which the interaction between CNTs and adsorbed $\mathrm{NH}_{3}$ gas is increased as gas concentration is increased. However, when the gas concentration is further increased, some $\mathrm{NH}_{3}$ molecules are adsorbed on the CNT sidewall, the other molecules must diffuse into the CNT film to find available sites. Therefore, the sensitivity increases slowly in this region.

The CNTs can be functionalized with nanoparticles or composite to improve their gas sensitivity. On the one hand, the functionalization of the CNTs involves the combination of the properties of the CNTs and nanoparticles in nanocomposite CNT/nanoparticle structures, which can exhibit new properties that differ from those of their individual parts. On the other hand, the nanotubes in gas sensing applications are decorated by the nanoparticles to provide selective sensitivity to different gases. Several studies have demonstrated that the CNTs can be decorated with Pd to increase the sensitivity [18], coated with Pt, Pd, Rh, and Au for the selectivity of gas sensor array [19], or sputtered with Ti for gas sensing at low temperature [20]. In this study, we used Co to decorate the obtained CNTs and investigate the effect of Co/CNT interaction on the sensitivity and selectivity of the sensor. The modification of the MWCNTs involves sputtering of $\sim 2 \mathrm{~nm}$ of Co on the entire substrate that contains the MWCNT-based sensor. The CNTs film is coated by very thin Co layer ( $2 \mathrm{~nm})$. It is revealed that the change in morphology is not clear (the corresponding SEM image is not shown here), except the tubes surface is rougher. The growth of this thin metal layer results in the formation of nanoparticles that decorate the sidewall of the nanotubes [18-20].

Figure 3 shows the responses of the sensor (MWCNTs were coated with Co nanoparticles) to $\mathrm{NH}_{3}$ gas at room temperature and at various gas concentrations (7, 14, 21, and $28 \mathrm{ppm}$, respectively). The responses of the sensor increased approximately two times compared with that of the uncoated samples. The sensor response cycles against the time of exposure to $\mathrm{NH}_{3}$ gas and dry air showed a good repeatability. The response time is approximately $30 \mathrm{~s}$ and the recovery time varies from $200 \mathrm{~s}$ to $500 \mathrm{~s}$, which depends on the $\mathrm{NH}_{3}$ gas concentration. Both the response and recovery times (before and after Co-coating) of our sensors are much shorter compared to some other works [9,13,21]. The increase in the recovery time of Co-coated devices could be explained by the presence of surface chemisorptions additional to physisorption in the case of uncoated samples. Meanwhile, the increase in sensor 
sensitivity can be caused by the metal/CNT interaction. A depletion region is formed when the CNT sidewall is coated with Co nanoparticles. The $\mathrm{NH}_{3}$ molecules donate electrons to the $\mathrm{CNT}$ sidewall, thereby expanding the depletion region, which reduces the carrier (holes) transport on the CNT wall and decreases the carrier mobility as well as the conductivity of the device. The sensor resistance is then increased.

Figure 3. Sensor response to $7 \mathrm{ppm}$ (a), $14 \mathrm{ppm}$ (b), $21 \mathrm{ppm}$ (c), and $28 \mathrm{ppm}$ (d) of $\mathrm{NH}_{3}$ on and off cycles. The surface of the device is coated with Co ( $2 \mathrm{~nm}$ thick) before gas exposure.

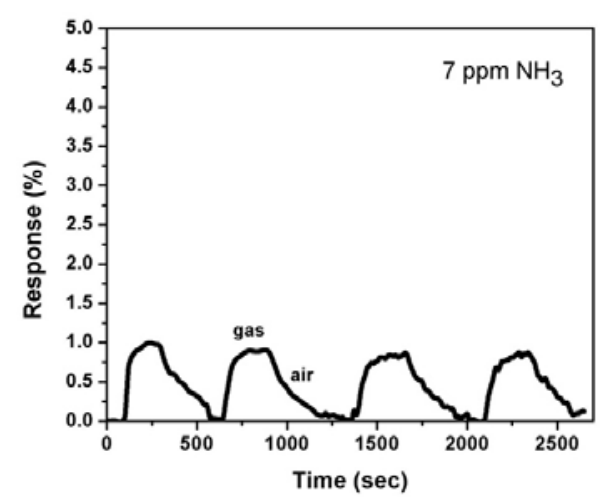

a)

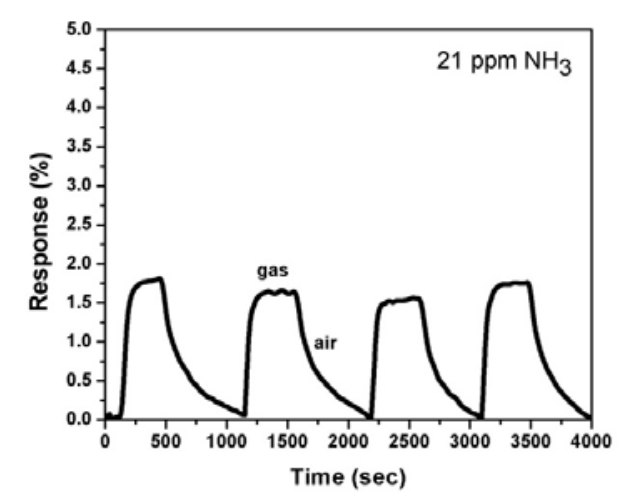

c)

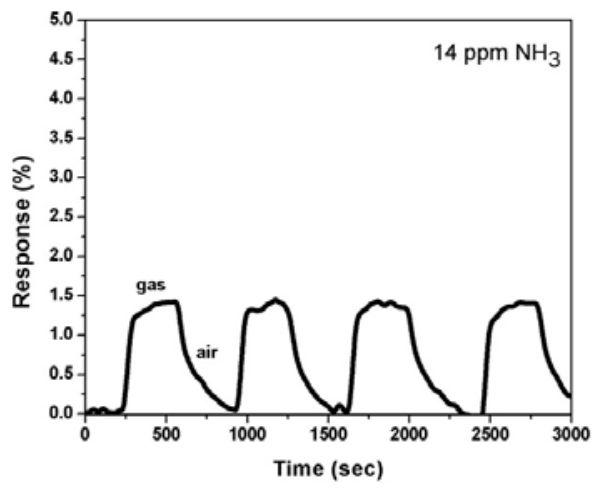

b)

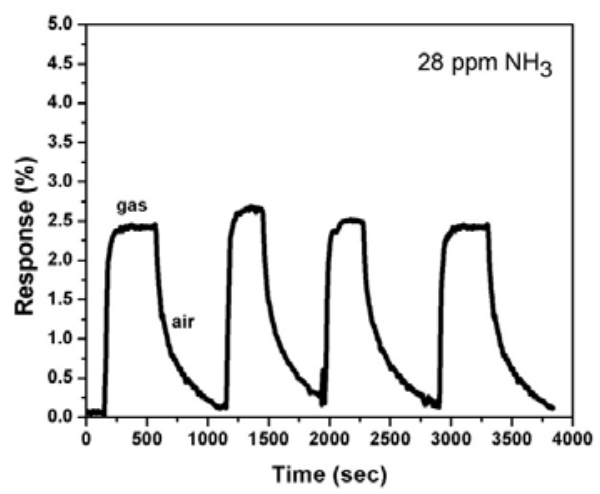

d)

Figure 4(a) shows the two cycles of sensor responses to $\mathrm{NH}_{3}$ gas at low concentrations of 28, 42, 56, and $70 \mathrm{ppm}$ (a step of $14 \mathrm{ppm}$ ). Figure 4(b) shows the sensor response to $\mathrm{NH}_{3}$ gas at high concentrations (up to $900 \mathrm{ppm}$ ). The sensor response increased by a factor of two compared with that in Figure 2(a,b). This result is consistent with that in Figure 3, which shows that the increase in response is caused by the expansion of the combined depletion layers of metal/CNTs and $\mathrm{NH}_{3}$ molecules/CNTs on the CNT sidewall.

The relationship between the sensor responses and $\mathrm{NH}_{3}$ concentration is illustrated in Figure 4(c), which is similar to the result obtained for the samples uncoated with metal nanoparticles Figure 2(c). The responses in the low concentration region increased more rapidly than that in the high concentration region. The decrease in the slope is due to the limit of adsorption sites on the MWCNT sidewall on the sample surface.

Figure 4(d) shows the responses of the sensor to $\mathrm{NH}_{3}$ gas compared with alcohol and $\mathrm{LPG}$. $\mathrm{NH}_{3}$, alcohol, and LPG concentrations were 7, 180, and 900 ppm, respectively. These concentrations were chosen to observe clearly the different sensor response curves towards these three types of gases. The 
sensor responded to $\mathrm{NH}_{3}$ as soon as the gas was injected. By contrast, the sensor was almost insensitive to alcohol and LPG. The same results were also obtained at higher gas concentrations. Thus, our MWCNT based sensor demonstrated high selectivity to $\mathrm{NH}_{3}$ gas at room temperature.

Figure 4. Responses of the MWCNT based sensor decorated with Co nanoparticles to $\mathrm{NH}_{3}$ gas at low (a) and high (b) gas concentration; Dependence of the responses on $\mathrm{NH}_{3}$ concentration (c); Comparison of the sensor responses to $\mathrm{NH}_{3}$, alcohol, and LPG which demonstrated that the sensor was selective to $\mathrm{NH}_{3}(\mathbf{d})$.

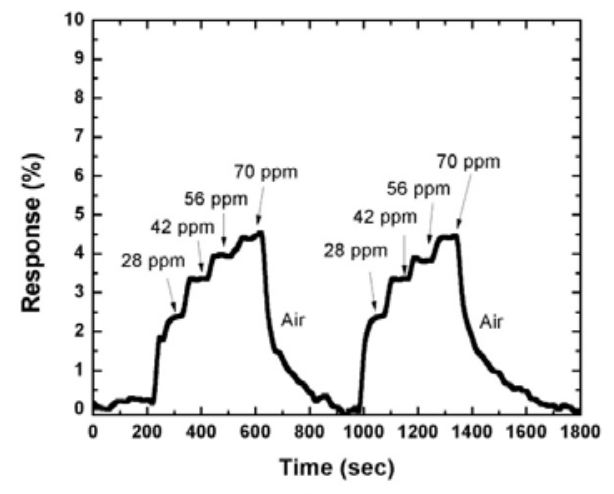

a)

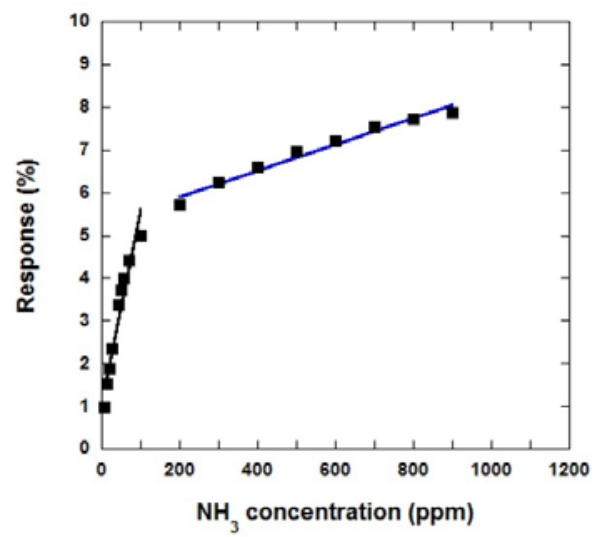

c)

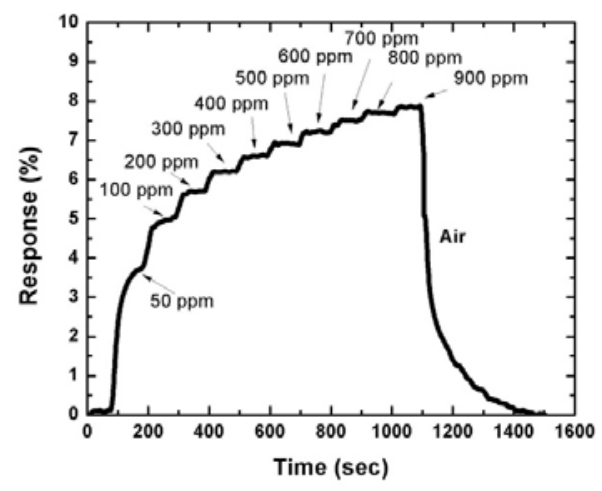

b)

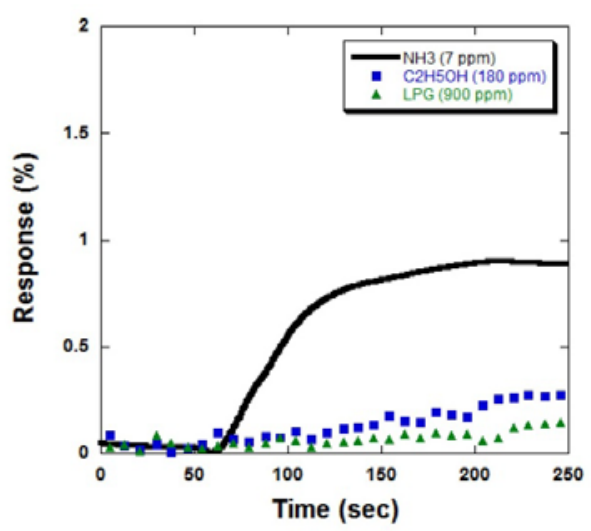

d)

\section{Conclusions}

MWCNTs were successfully grown on the alumina substrate with two Pt electrodes to fabricate a gas sensor. The sensor was sensitive to $\mathrm{NH}_{3}$ gas at a wide range of concentrations from some ppm to $900 \mathrm{ppm}$. Co nanoparticles were decorated on the CNT sidewall to improve both the sensitivity and selectivity of the sensors.

\section{Acknowledgments}

The authors would like to thank the support from Vietnam's National Foundation for Science and Technology Development (NAFOSTED) project, code 103.02.99.09. 


\section{References}

1. Devi, G.S.; Subrahmanyam, V.B.; Gadkari, S.C.; Gupta, S.K. $\mathrm{NH}_{3}$ gas sensing properties of nanocrystalline $\mathrm{ZnO}$ based thick films. Anal. Chem. Acta 2006, 568, 41-46.

2. Thong, L.V.; Loan, L.T.N.; Hieu, N.V. Comparative study of gas sensor performance of $\mathrm{SnO}_{2}$ nanowires and their hierarchical nanostructures. Sens. Actuators B 2010, 150, 112-119.

3. Huang, J.R.; Wang, J.H.; Gu, C.P.; Yu, K.; Meng, F.L.; Liu, J.H. A novel highly sensitive gas ionization sensor for ammonia detection. Sens. Actuators A 2009, 150, $218-223$.

4. Chen, Y.; Menga, F.L.; Li, M.Q.; Liu, J.H. Novel capacitive sensor: Fabrication from carbon nanotube arrays and sensing property characterization. Sens. Actuators B 2009, 140, 396-401.

5. Suehiro1, J.; Zhou, G.; Hara, M. Fabrication of a carbon nanotube-based gas sensor using dielectrophoresis and its application for ammonia detection by impedance spectroscopy. J. Phys. D Appl. Phys. 2003, 36, L109-L114.

6. Paolo, B.; Pierre, L.; Didier, P. Carbon nanotubes based transistors as gas sensors: State of the art and critical review. Sens. Actuators B 2009, 140, 304-318.

7. Chopra, S.; Pham, A.; Gaillard, J.; Parker, A.; Rao, A.M. Carbon-nanotube-based resonant-circuit sensor for ammonia. Appl. Phys. Lett. 2002, 80, 4632-4634.

8. Radouane, L.; Roman, P.; Alexandre, F.; Alexey, V.; Carles, C.; Isabel, G.; Jean-Jacques, P.; Eduard, L. Gas sensors based on multiwall carbon nanotubes decorated with tin oxide nanoclusters. Sens. Actuators B 2010, 145, 411-416.

9. Sharma, S.K.; Sengupta, S.; Islam, S. Deposition of Pristine and functionalized MWCNTs in alumina matrix by sol-gel technique and investigation of their ammonia sensing properties. Nanomater. Nanotechnol. 2012, 2, 1-6.

10. Kaneto, K.; Tsuruta, M.; Sakai, G.; Cho, W.Y.; Ando, Y. Electrical conductivities of multi-wall carbon nanotubes. Synth. Met. 1999, 103, 2543-2546.

11. Varghese, O.K.; Kichambre, P.D.; Gong, D.; Ong, K.G.; Dickey, E.C.; Grimes, C.A. Gas sensing characteristics of multi-wall carbon nanotubes. Sens. Actuators B 2001, 81, 32-41.

12. Nguyen, V.H.; Luong, T.B.T.; Nguyen, D.C. Highly sensitive thin film $\mathrm{NH}_{3}$ gas sensor operating at room temperature based on $\mathrm{SnO}_{2} / \mathrm{MWCNTs}$ composite. Sens. Actuators B 2008, 129, 888-895.

13. Arab, M.; Berger, F.; Picaud, F.; Ramseyer, C.; Glory, J.; Mayne-L'Hermite, M. Direct growth of the multi-walled carbon nanotubes as a tool to detect ammonia at room temperature. Chem. Phys. Lett. 2006, 433, 175-181.

14. Wang, S.G.; Zhang, Q.; Yang, D.J.; Sellin, P.J.; Zhong, G.F. Multi-walled carbon nanotube-based gas sensors for NH detection. Diam. Relat. Mater. 2004, 13, 1327-1332.

15. Liu, H.Z.; Ma, H.; Zhou, W.; Liu, W.H.; Zheng, J.; Li, X. Synthesis and gas sensing characteristic based on metal oxide modification multi wall carbon nanotube composites. Appl. Surf. Sci. 2012, 258, 1991-1994.

16. Li, Y.H.; Zhao, Y.M.; Zhu, Y.Q.; Rodriguez, J.; Morante, J.R.; Mendoza, E.; Poa, C.H.P.; Silva, S.R.P. Mechanical and $\mathrm{NH}_{3}$ sensing properties of long multi-walled carbon nanotube ropes. Carbon 2006, 44, 1821-1825.

17. Faizah, M.Y.; Fakhru'l-Razi, A.; Sidek, R.M.; Liew, A.A.G. Gas sensor application of carbon nanotubes. Int. J. Eng. Technol. 2007, 4, 106-113. 
18. Kong, J.; Chapline, M.G.; Dai, H. Functionalized carbon nanotubes for molecular hydrogen sensors. Adv. Mater. 2001, 13, 1384-1386.

19. Star, A.; Joshi, V.; Skarupo, S.; Thomas, D.; Gabriel, J.C.P. Gas sensor array based on metal-decorated carbon nanotubes. J. Phys. Chem. B 2006, 110, 21014-21020.

20. Gelamo, R.V.; Rouxinol, F.P.; Verissimo, C.; Vaz, A.R.; de Moraes, M.A.; Moshkalev, B.S.A. Low-temperature gas and pressure sensor based on multi-wall carbon nanotubes decorated with Ti nanoparticles. Chem. Phys. Lett. 2009, 482, 302-306.

21. Ionescu, R.; Espinosa, E.H.; Sotter, E.; Llobet, E.; Vilanova, X.; Correig, X.; Felten, A.; Bittencourt, C.; Van Lier, G.; Charlier, J.-C.; et al. Oxygen functionalisation of MWCNT and their use as gas sensitive thick-film layers. Sens. Actuators B 2006, 113, 36-46.

(C) 2013 by the authors; licensee MDPI, Basel, Switzerland. This article is an open access article distributed under the terms and conditions of the Creative Commons Attribution license (http://creativecommons.org/licenses/by/3.0/). 